Modern Physics Letters A

(C) World Scientific Publishing Company

\title{
Schrödinger Equation of a particle in an Uniformly Accelerated Frame and the Possibility of a New kind of Quanta
}

\author{
Sanchari De ${ }^{a}$ \\ ${ }^{a}$ Department of Physics, Visva-Bharati, Santiniketan, India 731235 \\ E-mail: sancharide19@gmail.com \\ Sutapa Ghosh ${ }^{b}$ \\ ${ }^{b}$ Department of Physics, Barasat Govt. College, Barasat 700124, India \\ E-mail:neutronstar12@yahoo.co.in \\ Somenath Chakrabarty ${ }^{a}$ \\ ${ }^{a}$ Department of Physics, Visva-Bharati, Santiniketan, India 731235 \\ E-mail: somenath.chakrabarty@visva-bharati.ac.in
}

Received (Day Month Year)

Revised (Day Month Year)

\begin{abstract}
In this article we have developed a formalism to obtain the Schrödinger equation for a particle in a frame undergoing an uniform acceleration in an otherwise flat Minkowski space-time geometry. We have presented an exact solution of the equation and obtained the eigenfunctions and the corresponding eigenvalues. It has been observed that the Schrödinger equation can be reduced to an one dimensional hydrogen atom problem. Whereas, the quantized energy levels are exactly identical with that of an one dimensional quantum harmonic oscillator. Hence considering transitions, we have predicted the existence of a new kind of quanta, which will either be emitted or absorbed if the particles get excited or de-excited respectively.
\end{abstract}

Keywords: Uniformly accelerated motion; Rindler coordinates; Fowler-Nordheim emission.

PACS Nos.: 03.65.Ge,03.65.Pm,03.30.+p,04.20.-q

In the case of inertial frame of references, in the relativistic scenario, the set of space-time coordinates are transformed following the well known Lorentz formulas. ${ }^{[1] 2}$ However, for a frame undergoing an uniform accelerated motion in an otherwise flat Minkowski space-time geometry, the Lorentz type transformation can also be derived to relate the coordinate sets in accelerated frame with that of inertial set of coordinates. $\frac{\sqrt[3]{5}}{5}$ These are the so called Rindler coordinate transformations and 
are given by:

$$
\begin{aligned}
c t & =\left(\frac{c^{2}}{\alpha}+x^{\prime}\right) \sinh \left(\frac{\alpha t^{\prime}}{c}\right) \text { and } \\
x & =\left(\frac{c^{2}}{\alpha}+x^{\prime}\right) \cosh \left(\frac{\alpha t^{\prime}}{c}\right)
\end{aligned}
$$

Hence one can also express the inverse relations

$$
c t^{\prime}=\frac{c^{2}}{2 \alpha} \ln \left(\frac{x+c t}{x-c t}\right) \text { and } x^{\prime}=\left(x^{2}-(c t)^{2}\right)^{1 / 2}-\frac{c^{2}}{\alpha}
$$

Where $\alpha$ is the uniform acceleration of the frame. The Rindler space-time coordinates as shown above (eqns.(1) and (2)) are then just an accelerated frame transformation of the Minkowski metric of special relativity.

We know that in the case of Schrödinger equation, the space time geometry is essentially flat and Euclidean in nature. On the other hand, in the relativistic picture, in the study of quantum mechanical equations, e.g., Klein-Gordon equation or Dirac equation, the geometry is also flat, but Minkowski type. In the curved space-time configuration, where the geometry is Riemann type, i.e., the formalism is based on the general theory of relativity, a substantial amount of work have already been done on the study of both Klein-Gordon equation ${ }^{6}$ and Dirac equation ${ }^{7}$ (see $\operatorname{alsc}^{3 \sqrt[3]{8}}$. $)$. However, so far our knowledge is concerned nothing has been reported in the literature on the study of Schrödinger equation in uniformly accelerated frame with non-relativistic approximation.

In this article we have developed a formalism to obtain the Schrödinger equation in an uniformly accelerated frame, or in otherwise speaking, in accordance with the principle of equivalence in a rest frame in presence of a constant gravitational field. Our study is thus based on the principle of equivalence, according to which a frame of reference undergoing an accelerated motion in absence of a gravitational field is equivalent to a frame at rest in presence of a gravitational field. To the best of our knowledge the study of Schrödinger equation in uniformly accelerated frame has not been done before.

Now it can very easily be shown that under Rindler coordinates transformation the Minkowski line element changes from ${ }^{5}$

$$
d s^{2}=d(c t)^{2}-d x^{2}-d y^{2}-d z^{2} \text { to } d s^{2}=\left(1+\frac{\alpha x^{\prime}}{c^{2}}\right)^{2} d\left(c t^{\prime}\right)^{2}-d x^{\prime 2}-d y^{\prime 2}-d z^{\prime 2}
$$

Now following the formalism of relativistic dynamics of special theory of relativity, the action integral can be written as ${ }^{1}$

$$
S=-\alpha_{0} \int_{a}^{b} d s \equiv \int_{a}^{b} L d t
$$

Then following ${ }^{1}$ after putting $\alpha_{0}=-m_{0} c$, where $m_{0}$ is rest mass of the particle and finally omitting the prime symbols, the Lagrangian of the particle can be written 
$a \sqrt{5}$

$$
L=-m_{0} c^{2}\left[\left(1+\frac{\alpha x}{c^{2}}\right)^{2}-\frac{v^{2}}{c^{2}}\right]^{1 / 2}
$$

To develop the non-relativistic version of quantum mechanical formalism for a particle in an uniformly accelerated frame, we start with this single particle classical Lagrangian in Rindler space. Here $v$ the particle velocity along $x$-direction. Then assuming three dimensional motion, the three momentum vector of the particle may be written as

$$
\vec{p}=\frac{m_{0} \vec{v}}{\left[\left(1+\frac{\alpha x}{c^{2}}\right)^{2}-\frac{v^{2}}{c^{2}}\right]^{1 / 2}}
$$

Obviously we get back the special theory result for $\alpha=0$. Hence the classical Hamiltonian of the particle is given by

$$
H=m_{0} c^{2}\left(1+\frac{\alpha x}{c^{2}}\right)\left(1+\frac{p^{2}}{m_{0}^{2} c^{2}}\right)^{1 / 2}
$$

Which also reduces to the Hamiltonian of special theory of relativity for $\alpha=0$. Now in the quantum mechanical picture, the classical dynamical variables $x, \vec{p}$ and also $H$ are treated as operators, with the canonical commutation relations

$$
\left[x, p_{x}\right]=i \hbar \text { and }\left[x, p_{y}\right]=\left[x, p_{z}\right]=0
$$

The Schrödinger equation for the particle is then given by

$$
H \psi=\left(1+\frac{\alpha x}{c^{2}}\right)\left(m_{0} c^{2}+\frac{p^{2}}{2 m_{0}}\right) \psi=E \psi
$$

Here the origin of co-ordinate is assumed to be at the centre of a strongly gravitating object, say a black hole and $x$ is measured along the positive direction. Now considering the eigenvalue form of the operator $1+\alpha x / c^{2}$ on the right hand side, we have

$$
\left(m_{0} c^{2}+\frac{p^{2}}{2 m_{0}}\right) \psi=\frac{1}{1+\frac{\alpha x}{c^{2}}} E \psi
$$

Since there is an $x$-dependent term in the right hand side of the above equation, the separable form of solution for the Schrödinger equation can obviously be written as

$$
\psi(x, y, z)=N X(x) \exp \left(-\frac{i p_{y} y}{\hbar}\right) \exp \left(-\frac{i p_{z} z}{\hbar}\right)
$$

where $X(x)$ is some unknown function of $x$. On substituting $\psi(x, y, z)$ in eqn.(10), we have

$$
\left(m_{0} c^{2}+\frac{p_{y}^{2}}{2 m_{0}}+\frac{p_{z}^{2}}{2 m_{0}}\right) X-\frac{\hbar^{2}}{2 m_{0}} \frac{d^{2} X}{d x^{2}}=\frac{1}{1+\frac{\alpha x}{c^{2}}} E X
$$


Now defining the quantity within the first bracket on the left hand side as $E_{\perp}$, the orthogonal part of the total energy, including the rest mass energy of the particle, we have

$$
E_{\perp} X-\frac{\hbar^{2}}{2 m_{0}} \frac{d^{2} X}{d x^{2}}=\frac{1}{1+\frac{\alpha x}{c^{2}}} E X
$$

Let us now introduce a new variable $u=1+\alpha x / c^{2}$ and define two constant quantities

$$
a=\frac{2 m_{0} c^{4}}{\hbar^{2} \alpha^{2}} E \text { and } b=\frac{8 m_{0} c^{4}}{\hbar^{2} \alpha^{2}} E_{\perp}
$$

then we can express the above equation (eqn.(13)) in the following form

$$
\frac{d^{2} X}{d u^{2}}+\frac{a}{u} X-\frac{b}{4} X=0
$$

Again defining $w=b^{1 / 2} u$ as a new variable and $\gamma=a b^{-1 / 2}$ another constant quantity, we have from the above equation

$$
\frac{d^{2} X}{d w^{2}}+\left(-\frac{1}{4}+\frac{\gamma}{w}\right) X=0
$$

On comparing this differential equation with that satisfied by the Whittaker func-

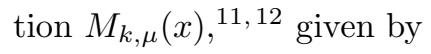

$$
\frac{d^{2}}{d x^{2}} M_{k, \mu}(x)+\left(-\frac{1}{4}+\frac{k}{x}+\frac{\frac{1}{4}-\mu^{2}}{x^{2}}\right) M_{k, \mu}(x)=0
$$

we have $X(x) \equiv M_{k, \mu}(x)$, for $x=w, \mu=1 / 2$ and $k=\gamma$. Then one can write down the solution in the explicit form as

$$
X(w)=M_{\gamma, \frac{1}{2}}(w)=\exp \left(-\frac{w}{2}\right) w M(1-\gamma, 2, w)
$$

where $M(a, c, x)={ }_{1} F_{1}(a ; c ; x)$, the Confluent Hypergeometric function. From the properties of special functions, clearly, $M(1-\gamma, 2, w)$ will be a polynomial and becomes zero for $w \longrightarrow \infty$, if the parameter $1-\gamma$ is 0 or a negative integer, i.e., $\gamma=n$, for $n=1,2,3 \ldots \ldots$. the positive integers. This is the physical condition to have a bounded wave function along $x$-direction. Under such restricted situation, the solution can also be expressed in terms of Associated Laguerre function. This alternative form of wave function is then given by

$$
X(w)=\exp \left(-\frac{w}{2}\right) w L_{\gamma-1}^{1}(w)
$$

The parameter $\gamma$ is again have to be non-zero positive integer. It can very easily be verified that the form of eqn(15) is exactly identical with the equation for an one-dimensional hydrogen atom. 13$] 14$ Then it is just a matter of simple algebra to show from the expression for $\gamma$ that this restriction gives quantized form of energy of the particle in an uniformly accelerated frame. Using the expressions for $a, b$ and 
$E_{\perp}$, it is straight forward to show that the quantized form of energy of the particle is given by

$$
E_{n}=n \hbar \frac{\alpha}{c}=n \hbar \omega \quad \text { (say) }
$$

with

$$
\omega=\frac{\alpha}{c}
$$

where we have put $2^{1 / 2} \approx 1$ for aesthetic sense and neglected $p_{y}$ and $p_{z}$ for a purely one-dimensional condition. Then accordingly $E_{\perp}=m_{0} c^{2}$, the rest mass energy of the particle. Therefore it is quite surprising result. The differential equation satisfied by the particle is exactly identical with the equation for an one-dimensional hydrogen atom, whereas the quantized energy levels are exactly look like that of the energy levels for an one-dimensional quantum harmonic oscillator.

Therefore we may conclude, by saying that in an uniformly accelerated frame or in Rindler space, the Schrödinger equation for a particle reduces to the identical form of differential equation satisfied by an one-dimensional hydrogen atom. Further, the restriction imposed on the solution, to make it physically acceptable, gives the quantized energy levels. The energy levels are found to be exactly identical with that of one-dimensional quantum harmonic oscillator. The energy levels vary linearly with the quantum number $n$, instead of $1 / n^{2}$, where the last one is the case for an one-dimensional hydrogen atom. The energy levels are observed to be independent of particle rest mass. It depends only on the acceleration $\alpha$ of the frame. It is evident from eqn.(19) that $\omega \longrightarrow 0$ as $\alpha \longrightarrow 0$. Further, unlike the one-dimensional quantum mechanical harmonic oscillator, the minimum energy of the particle or the ground state energy for a given $\alpha$ is $\hbar \omega$ for $n=1$, i.e., there is no zero point energy. It is also obvious from the analysis that the energy levels are produced by the uniform gravitational field or the constant acceleration of the frame. Therefore if any transition takes place from some higher to lower energy levels, the emitted energy will not be of any kind of conventional or known type quanta. We call it as the dark quanta or the cosmic phonon and the dark wave for its classical counter part. In the case of excitation to some higher energy levels the absorbed energy must also be in the form of dark quanta.

Now from eqn.(20) one can also write $\lambda \alpha=$ constant, which is the gravitational form of Wien's displacement law (for the usual black body case it is $\lambda T=$ constant, where $T$ is the temperature of the black body system). To elaborate this point a little more, we assume that if a large number of cosmic phonons are created in the very early universe, within a region where the gravitational field is uniform, even before the epoch when the matter and radiation are not decoupled, then as the universe expands, since the gravitational field decreases, the wave length of this non-thermal cosmic phonon field will increase. Therefore, the gravitational field $\alpha$ plays the role of $T$ for a thermal field in equilibrium, e.g., the CMBR. Therefore based on our model calculation we may assume that the non-thermal phonon field, which may also be assumed to be some kind of neutral scalar field and the thermal field CMBR 
exist side by side. Whether they can interact with each other is a matter of further investigation. Further, since the energy levels are created in presence of background uniform gravitational field, the concept of spin of the emitted or absorbed phonons are not considered here. Again defining some kind of refractive index $\mu \propto \alpha$, we have $\mu \lambda=$ constant. Since the gravitational field at the present epoch is low enough, assuming Newtonian gravity, we may write

$$
\alpha \propto \frac{1}{x_{l}}
$$

where $x_{l}$ is the position of the local rest frame, then

$$
\lambda \propto x_{l}^{2}
$$

Therefore the wavelength decreases as the square of some length parameter. Further, it is quite possible that a large number of such cosmic phonons might have produce at the proximity of supper massive black holes present at the centre of the galaxies. However, in this case since there is no expansion, these cosmic phonons or the dark quanta or the classical counter part, the dark waves remain confined at the vicinity of massive black holes. Therefore it is quite likely that these waves or quanta could be treated as one of the viable candidates for dark matter. Of course at present we can not give any experimental technique to detect these cosmic phonons as dark matter.

In some separate analysis, $\frac{15}{15}$ keeping only the linear term in $x$ in the expansion of $\left(1+\alpha x / c^{2}\right)^{-1}$, we have noticed that the Schrödinger equation can be reduced to a differential form, which is identical with Fowler-Nordheim equation ${ }^{16}$ for the field emission or cold emission of electrons from a metal surface under the action of a strong electric field applied near the metal surface. In that analysis we have observed, that particle production near the event horizon of a black hole, in the non-relativistic approximation is almost identical with the phenomena of FowlerNordheim emission.

Further, keeping up to the quadratic term in $x$, we have also got an analytical solution for the Schrödinger equation, which is the parabolic cylindrical function. However, it has no physical significance.

In our final conclusion, we would like to mention that further study is needed to know the spin, parity etc. for the emitted or absorbed dark quanta if they really exist.

\section{References}

1. Landau L.D. and Lifshitz E.M., The Classical Theory of Fields, ButterworthHeimenann, Oxford, (1975).

2. W.G. Rosser, Contemporary Physics, 1, 453, (1960).

3. N.D. Birrell and P.C.W. Davies, Quantum Field Theory in Curved Space, Cambridge University Press, Cambridge, (1982).

4. C.G. Huang and J.R. Sun, arXiv:gr-qc/0701078, (2007).

5. Domingo J Louis-Martinez, Class. Quantum Grav., 28, 036004, (2011). 
6. D. Percoco and V.M. Villaba, Class. Quantum Grav., 9, 307, (1992).

7. Nicola Vona, Master Thesis in Physics, University of Naples Federico II, (2007).

8. B.J.B Crowley et. al., Scientific Report — 2:491 —, DOI: 10.1038/srep00491.

9. Shahen Haeyan, arXiv:1105.0631v1.

10. M. Soffel, B. Müller and W. Greiner, Phys. Rev. D22, 1935, (1980).

11. M. Abramowitz and I.A. Stegan ed. Handbook of Mathematical Functions, Dovar Publications INC. (1970).

12. G.B. Arfken, H.J. Weber and F.E. Harris, Mathematical methods for Physicists, A Comprehensive guide, Seventh Edison, Elsevier, Lomdon, (2013).

13. Y. Ran, L. Xue, S. Hu and Ru-Keng Su, Jour. Phys. S:Math. Gen., 33, 9265, (2000).

14. G. Palma and U. Raff, Can. Jour. Phys., 84, 787, (2006).

15. S. De, S. Ghosh and S. Chakrabarty, arXiv:1412.6893.

16. R. H. Fowler and Dr. L. Nordheim, Proc. R. Soc. London 119, 173, (1928). 